\title{
Satisfação do paciente e acompanhante quanto ao atendimento de necessidades de cuidados de enfermagem*
}

\author{
Priscila Fernandes Martins ${ }^{1}$, Marcia Galan Perroca ${ }^{2}$
}

\author{
* Parte da dissertação de mestrado \\ intitulada "Percepção e nível de satisfação \\ do paciente/acompanhante com o \\ atendimento de necessidades de cuidados" \\ defendida no Programa de Pós-Graduação \\ em Enfermagem da Faculdade de Medicina \\ de São José do Rio Preto, em 2016. Estudo \\ vinculado ao grupo de pesquisa Gestão de \\ Serviços de Saúde e de Enfermagem \\ (GESTSAÚDE). \\ ${ }^{1}$ Enfermeira, Mestre em Enfermagem. \\ Enfermeira do Hospital do Coração do \\ Instituto de Moléstias Cardiovasculares. \\ São José do Rio Preto, SP, Brasil. E-mail: \\ fernandespri@hotmail.com. \\ ${ }^{2}$ Enfermeira, Doutora em Enfermagem. \\ Professora Adjunta da Faculdade de \\ Medicina de São José do Rio Preto. São \\ José do Rio Preto, SP, Brasil. E-mail: \\ marcia.perroca@gmail.com.
}

Recebido: 04/05/2016.

Aceito: 20/04/2017.

Publicado: 16/08/2017.

\section{Como citar esse artigo:}

Martins PF, Perroca MG. Satisfação do paciente e acompanhante quanto ao atendimento de necessidades de cuidados de enfermagem. Rev. Eletr. Enf. [Internet]. 2017 [acesso em:_____ ] _ 19:a18. Disponível em:

http://dx.doi.org/10.5216/ree.v19.41138.

\section{RESUMO}

Estudo descritivo-exploratório conduzido junto a 411 pacientes e seus acompanhantes com o propósito de examinar a percepção e nível de satisfação de ambos quanto ao atendimento das necessidades de cuidados durante o período de hospitalização e, também, verificar a associação deste nível com variáveis sociodemográficas e características da hospitalização e da unidade. Na coleta de dados foi utilizado instrumento autoadministrado. O escore de satisfação dos pacientes/acompanhantes variou de $3,6(0,4)$ a $4,6(0,4)$. As necessidades emocionais e espirituais ( 81 e $82 \%$ ), segurança (83 e $67 \%$ ) e atenção (87 e 61\%) foram as mais atendidas. Encontrou-se associação entre satisfação e algumas variáveis sociodemográficas e características da hospitalização. De maneira geral, os pacientes e seus acompanhantes mostraram-se satisfeitos com o atendimento das necessidades de cuidados, porém algumas áreas importantes apresentaram déficit de satisfação demandando maior atenção da equipe de enfermagem.

Descritores: Satisfação do Paciente; Determinação de Necessidades de Cuidados de Saúde; Cuidados de Enfermagem; Qualidade da Assistência à Saúde.

\section{INTRODUÇÃO}

O cuidado centrado no paciente consiste no reconhecimento, por parte dos profissionais, da singularidade e dos valores dos clientes considerando suas características pessoais, condições clínicas, situação de vida pessoal e respeitando suas preferências na participação da assistência ${ }^{(1-2)}$. Com foco central no paciente/família, sua realização apoia-se na busca da qualidade das relações pessoais, profissionais e organizacionais ${ }^{(3)}$. 
O acompanhante ou familiar representa presença positiva durante o período de permanência do paciente no hospital visto que contribui para o bem-estar mental, físico, social e espiritual deste e compartilha algumas atividades de trabalho com a equipe de enfermagem ${ }^{(4)}$. A aprovação de leis e decretos que regulamentam o direito à permanência de acompanhante para alguns grupos específicos tem permitido a humanização do ambiente hospitalar.

Para que o cuidado seja qualificado como individualizado torna-se necessário que o paciente expresse suas necessidades, preferências e percepções durante sua interação com a equipe, e esta, por sua vez, ajuste suas intervenções de acordo com as preocupações de cuidados de saúde do paciente e as características físicas e socioambientais do contexto assistencial ${ }^{(5)}$.

Essa modalidade de cuidado é considerada parte da prática de enfermagem e sua realização impacta positivamente no resultado da assistência ${ }^{(6)}$. Contudo, o ambiente de saúde e suas rápidas mudanças tem afetado a habilidade da equipe em atender as necessidades de cuidado de enfermagem dos pacientes. Expressivo número de enfermeiros tem relatado falta de tempo hábil para completar atividades de cuidados $^{(7)}$. Necessidades de cuidados não satisfeitas servem como indicador da qualidade do cuidado de enfermagem, ou seja, o que os enfermeiros fazem ou deixam de fazer influenciam nos resultados do processo de cuidar ${ }^{(8)}$.

A qualidade percebida é o resultado da comparação que os clientes fazem entre as suas expectativas sobre um serviço e a sua percepção do modo como o serviço foi executado ${ }^{(9)}$. O cuidado realizado pelo enfermeiro é visto como o fator mais importante para sua avaliação ${ }^{(10)}$. A satisfação, um dos indicadores da qualidade, pode ser entendida como o nível em que os cuidados de enfermagem atendem às expectativas do paciente e encontra-se associada a vários aspectos, dentre eles, relacionamento enfermeiro/paciente, apoio afetivo, informações sobre a saúde, controle da decisão pelo paciente e a competência técnica do profissional que o assiste ${ }^{(11)}$. A importância da temática satisfação do paciente com o cuidar em enfermagem tem gerado estudos nacionais ${ }^{(11-15)} \mathrm{e}$ internacionais ${ }^{(6-7,16-17)}$.

As informações sobre o conjunto de percepções relacionados à qualidade da atenção recebida pelos pacientes têm sido utilizadas como uma estratégia nas instituições de saúde ${ }^{(17)}$. Para a equipe de enfermagem, elas possibilitam propostas de ações para melhoria da qualidade da assistência e implementação de mudanças na prática ${ }^{(11)}$.

Pesquisas de satisfação são conduzidas sob a ótica do paciente quanto ao atendimento de suas necessidades de cuidados de enfermagem. Este estudo se propõe a ampliar esta questão contemplando, também, a abordagem do acompanhante e correlacionando o nível de satisfação com diferentes variáveis. Visa responder às seguintes questões: De que forma os pacientes e seus acompanhantes percebem o atendimento de suas necessidades de cuidados pela equipe de enfermagem durante o período de hospitalização? Qual é o nível de satisfação de ambos? Há associação entre o nível de satisfação e variáveis sociodemográficas e características do hospital e da unidade? Para responder a estes questionamentos delineou-se este estudo objetivando examinar a percepção e o nível de satisfação de pacientes e 
acompanhantes quanto ao atendimento das necessidades de cuidados durante o período de hospitalização e verificar a associação deste nível com variáveis sociodemográficas e características da hospitalização e da unidade.

\section{MÉTODO}

Este estudo descritivo-exploratório de caráter transversal foi conduzido em unidades de clínicas médicas, cirúrgicas e especializadas (geriatria, doenças infecto-parasitárias (DIP), ginecologia, neurologia, ortopedia e traumatologia, oncologia e unidade de emergência) de duas instituições hospitalares $\left(H_{A}\right.$ e $\left.H_{B}\right)$ no interior do Estado de São Paulo. A primeira constitui-se em hospital de ensino público de 732 leitos e quadro de pessoal de enfermagem composto por 140 enfermeiros e 1.200 auxiliares/técnicos de enfermagem. O hospital B caracteriza-se como uma instituição filantrópica com 196 leitos e conta com 56 enfermeiros e 387 auxiliares/técnicos de enfermagem.

Para o cálculo do tamanho amostral foi utilizado o teste T como normalidade de um estudo piloto de tamanho 20x20, para diferença esperada entre as médias de 0,15 pontos, com desvio padrão estimado em 0,44 em cada hospital e poder 0,95 . Identificou-se que o tamanho mínimo estimado seria $\mathrm{N}=182$. Contudo, por segurança, foram investigados 200 pacientes.

Os participantes do estudo foram 411 pacientes adultos hospitalizados e seus acompanhantes ( $n=411)$. Os pacientes deveriam atender aos seguintes critérios:

1. Estarem sob tratamento nas unidades de internação e orientados no tempo e espaço;

2. Serem capazes de ler, entender e responder às questões do instrumento.

A inclusão do acompanhante ocorreu mediante atendimento do critério 2. A coleta de dados ocorreu no período de março a maio de 2014.

Foram investigadas variáveis:

1. Sociodemográficas (sexo, faixa etária, grau de instrução, ocupação e renda);

2. Características da hospitalização (hospital e tipo de internação, modalidade de internação, tempo de permanência do acompanhante junto ao paciente;

3. Características da unidade (turno de trabalho, quantidade de enfermeiros, técnicos e auxiliares de enfermagem e da equipe).

O estudo ocorreu em três etapas conforme descrição abaixo.

\section{Construção dos Instrumentos 1 e 2:}

- Devido à inexistência na literatura brasileira, até o presente momento, de um instrumento específico que atendesse aos objetivos do estudo e avaliasse a satisfação de ambos pacientes e acompanhantes sobre necessidades de cuidados foi construído um questionário. 0 conteúdo foi baseado na classificação das necessidades humanas básicas ${ }^{(18)}$, em estudo sobre auditoria da assistência de enfermagem ${ }^{(19)}$ e instrumento adaptado e validado no Brasil $^{(12-13)}$ dentre outros. 
- A primeira parte do questionário continha informações sociodemográficas do respondente. A outra foi composta por uma escala Likert de cinco pontos com opções variando de "concordo totalmente" a "discordo totalmente" sendo também incluída a alternativa "não se aplica". Foram elaboradas 34 afirmativas abrangendo 10 áreas de cuidados: Atenção; Informação; Segurança; Higiene e Conforto; Percepção Dolorosa e Terapêutica; Hidratação/Nutrição; Locomoção/Oxigenação/Regulação Térmica; Eliminações; Integridade Física e Pele e, Suporte Emocional e Espiritual.

- O segundo instrumento abrangia dados sobre as instituições hospitalares, unidades (número de leitos e pacientes) e equipes de enfermagem investigadas (turno de trabalho, quantidade de enfermeiros, técnicos e auxiliares de enfermagem);

\section{Validação do instrumento e estudo piloto:}

- Após a construção, o instrumento foi submetido à apreciação de cinco enfermeiros doutores e três enfermeiros clínicos para validação de seu conteúdo. Solicitou-se aos juízes considerações sobre objetividade, clareza e pertinência de cada uma das afirmações. Obteve-se concordância variando de 95 a 98\% evidenciando, dessa forma, que os itens propostos captavam adequadamente a satisfação de pacientes e acompanhantes sobre necessidades de cuidados. O questionário foi preenchido, preliminarmente, por 20 pacientes e seus acompanhantes. A consistência interna do instrumento revelou alfa de Cronbach de 0,70 considerado como satisfatória.

\section{Aplicação dos questionários através de entrevista:}

- Foi realizada por um dos pesquisadores após orientação dos participantes quanto ao conteúdo do instrumento utilizado e sua forma de operacionalização. Os pacientes e seus acompanhantes foram escolhidos aleatoriamente (sorteio a partir de uma listagem de pacientes internados).

- A coleta de informações somente foi iniciada após a autorização da administração dos hospitais, gerência de enfermagem e anuência dos participantes. O projeto foi aprovado pelo Comitê de Ética em Pesquisa da instituição campo de estudo (Parecer n²16.781/2013).

Para tratamento estatístico utilizou-se os programas Bioestat 5.3 e The R Foundation for Statistical Computing versão 2.13.0. O nível de significância adotado foi de 0,05. Considerou-se:

- A estatística descritiva apresentada como frequência, porcentagens, médias ( $M$ ) e desvio padrão (DP) para os dados sociodemográficos;

- A escala Likert como nível de mensuração ordinal sendo calculados mediana (Md) e amplitude interquartílica - AIQ (Q3 - Q1) para avaliação do nível de satisfação de pacientes e acompanhantes quanto ao atendimento das necessidades de cuidados;

- O percentual de concordância das respostas foi calculado por meio da somatória das respostas "4" concordo e " 5 " concordo totalmente, tendo como denominador o número total de pacientes ou 
acompanhantes entrevistados. Definiu-se concordância satisfatória como valores >60\%;

- Para associação do escore médio global de satisfação dos pacientes e acompanhantes com as variáveis sociodemográficas, características da hospitalização e da unidade foram utilizados o teste t (dois grupos) e Análise de Variância (ANOVA) (mais de dois grupos) com teste de Bonferroni com 95\% de intervalo de confiança.

\section{RESULTADOS}

Houve predomínio de pacientes do sexo masculino ( $n=125,62,5 \%)$ no HA com idade média de 52 (DP 17,6; variação 16-88) anos e ensino fundamental incompleto ( $n=113,56,5 \%)$. Seus acompanhantes eram, na sua maioria, do sexo feminino $(n=161,80,5 \%)$ apresentando idade média de 47 (DP 16; variação 18-80) anos e ensino médio completo $(n=87,43,5 \%)$.

No HB foram encontrados pacientes do sexo feminino ( $n=115,54,5 \%)$, com idade média de 51,7 (DP 17,4; variação 19-87) anos e ensino fundamental completo ( $n=71,33,6 \%)$. Desatacaram-se, igualmente, acompanhantes do sexo feminino ( $n=107,50,7 \%)$, com idade média de 48,3 (DP 17,2; variação 18-81) anos e ensino fundamental completo ( $n=108,51,2 \%)$. (Tabela 1$)$.

Tabela 1: Distribuição dos pacientes e acompanhantes segundo variáveis sociodemográficas no $H_{A}$ e $H_{B}(N=822)$ São José do Rio Preto, SP, Brasil, 2014.

\begin{tabular}{|c|c|c|c|c|}
\hline \multirow{3}{*}{ Variáveis } & \multicolumn{2}{|c|}{ Pacientes } & \multicolumn{2}{|c|}{ Acompanhantes } \\
\hline & $H_{A}(n=200)$ & $H_{B}(n=211)$ & $H_{A}(n=200)$ & $H_{B}(n=211)$ \\
\hline & $M(D P)$ & $N(\%)$ & $N(\%)$ & $N(\%)$ \\
\hline \multicolumn{5}{|l|}{ Sexo } \\
\hline Feminino & $75(37,5)$ & $115(54,5)$ & $161(80,5)$ & $107(50,7)$ \\
\hline Masculino & $125(62,5)$ & $96(45,5)$ & $107(50,7)$ & $104(49,3)$ \\
\hline \multicolumn{5}{|l|}{ Faixa etária (anos) } \\
\hline$<20$ & $7(3,5)$ & $4(1,9)$ & $8(4)$ & $4(1,9)$ \\
\hline $21-30$ & $18(9)$ & $25(11,8)$ & $36(18)$ & $37(17,5)$ \\
\hline $31-40$ & $40(20)$ & $38(18)$ & $32(16)$ & $38(18)$ \\
\hline $41-50$ & $24(12)$ & $31(14,7)$ & $40(20)$ & $40(20)$ \\
\hline $51-60$ & $39(19,5)$ & $38(18)$ & $36(18)$ & $27(12,8)$ \\
\hline$>61$ & $72(36)$ & $75(35,6)$ & $48(24)$ & $48(24)$ \\
\hline \multicolumn{5}{|l|}{ Grau de instrução } \\
\hline Fundamental incompleto & $113(56,5)$ & $64(30,3)$ & $69(34,5)$ & $34(16,1)$ \\
\hline Fundamental completo & $21(10,5)$ & $71(33,6)$ & $29(14,5)$ & $108(51,2)$ \\
\hline Médio completo & $57(28,5)$ & $70(33,1)$ & $87(43,5)$ & $62(29,4)$ \\
\hline Superior completo & $9(4,5)$ & $6(2,8)$ & $15(7,5)$ & $7(3,3)$ \\
\hline \multicolumn{5}{|l|}{ Ocupação } \\
\hline Ativo & $88(35)$ & $91(43,2)$ & $83(41,5)$ & $76(36)$ \\
\hline Afazeres domésticos & $35(17,5)$ & $33(15,6)$ & $70(35)$ & $44(20,8)$ \\
\hline Aposentado & $58(29)$ & $54(25,6)$ & $32(16)$ & $48(22,8)$ \\
\hline Desempregado & $19(9,5)$ & $33(15,6)$ & $15(7,5)$ & $43(20,4)$ \\
\hline \multicolumn{5}{|l|}{ Renda (salário mínimo) } \\
\hline$<1$ & $17(8,5)$ & $18(8,5)$ & $22(11)$ & $33(15,6)$ \\
\hline $1-3$ & $140(70)$ & $146(69,2)$ & $132(66)$ & $138(65,5)$ \\
\hline $3-5$ & $33(16,5)$ & $46(21,8)$ & $32(16)$ & $40(18,9)$ \\
\hline $5-10$ & $9(4,5)$ & $1(0,5)$ & $9(4,5)$ & - \\
\hline$>10$ & $1(0,5)$ & - & $9(4,5)$ & - \\
\hline
\end{tabular}


Encontrou-se maior percentual de concordância na satisfação dos pacientes referentes ao atendimento das necessidades emocionais e espirituais (81 e $82 \%$ ), segurança (83 e 67\%) e atenção (87 e $61 \%)$ em ambos os hospitais e informação (83\%) e percepção dolorosa e terapêutica (93\%) no $\mathrm{H}_{\mathrm{A}} \mathrm{Na}$ percepção dos acompanhantes foram as necessidades de segurança (91 e 67\%), percepção dolorosa e terapêutica (89 e 62\%), informação $\left(81 \% \mathrm{H}_{A}\right)$ e emocionais e espirituais $\left(76 \% \mathrm{H}_{B}\right)$ (Tabela 2$)$.

Tabela 2: Nível de satisfação dos pacientes e seus acompanhantes quanto ao atendimento de necessidades de cuidados ( $N=822)$. São José do Rio Preto, SP, Brasil, 2014.

\begin{tabular}{|c|c|c|c|c|c|c|c|c|}
\hline \multirow{4}{*}{ Áreas de cuidados } & \multicolumn{4}{|c|}{ Pacientes } & \multicolumn{4}{|c|}{ Acompanhantes } \\
\hline & \multicolumn{2}{|c|}{$H_{A}(n=200)$} & \multicolumn{2}{|c|}{$H_{B}(n=211)$} & \multicolumn{2}{|c|}{$H_{A}(n=200)$} & \multicolumn{2}{|c|}{$H_{B}(n=211)$} \\
\hline & Md & Conc & Md & Conc & Md & Conc & Md & Conc \\
\hline & AIQ & $\%$ & AIQ & $\%$ & AIQ & $\%$ & AIQ & $\%$ \\
\hline Atenção & $5(0)$ & 87 & $4(3)$ & 61 & $5(1)$ & 79 & $4(3)$ & 57 \\
\hline Informação & $5(1)$ & 83 & $3(2)$ & 49 & $5(1)$ & 81 & $4(3)$ & 53 \\
\hline Segurança & $5(0)$ & 83 & $4(2)$ & 67 & $5(0)$ & 91 & $4(2)$ & 67 \\
\hline Higiene e conforto & $2(5)$ & 46 & $3(3)$ & 46 & $3(5)$ & 48 & $4(4)$ & 57 \\
\hline Percepção dolorosa e terapêutica & $5(0)$ & 93 & $3(2)$ & 48 & $5(0)$ & 89 & $4(3)$ & 62 \\
\hline Hidratação/Nutrição & $2(0)$ & 21 & $4(5)$ & 0,5 & $2(4)$ & 54 & $4(5)$ & 55 \\
\hline Loco, Oxig e Regul térmica & $3(5)$ & 49 & $4(5)$ & 51 & $4(5)$ & 52 & $3(5)$ & 44 \\
\hline Eliminações & $1(0)$ & 18 & $2(0)$ & 0,4 & $1(3)$ & 35 & $1(5)$ & 55 \\
\hline Integridade Física e pele & $2(0)$ & 8 & $2(4)$ & 34 & $1(2)$ & 16 & $1(5)$ & 0,4 \\
\hline Emocionais e Espirituais & $5(0)$ & 81 & $5(1)$ & 82 & $5(0)$ & 16 & $5(1)$ & 76 \\
\hline
\end{tabular}

Loco, Oxig e Regul térmica = Locomoção, oxigenação e regulação térmica; Conc-concordância; AIQ=amplitude interquartílica (Q3 - Q1); Escore varia de 1 a 5; quanto maior o escore, mais alta é a concordância.

A associação entre o escore global e as variáveis sociodemográficas dos pacientes e acompanhantes mostrou-se significante $(p<0,05)$ para hospital investigado $\left(H_{A}\right.$ e $\left.H_{B}\right)$, sexo, grau de instrução, renda mensal, tipo e modalidade de internação e tempo de permanência junto ao paciente. Contudo, as diferenças ocorreram entre os hospitais e não intrahospitais (Tabela 3). Não foram encontradas interação entre o escore global com idade $(p=0,23)$ e religião $(p=0,64)$.

A associação entre o escore global médio de satisfação e as características das unidades onde os pacientes se encontravam internados (turnos e número de profissionais na equipe/categoria) encontram-se apresentadas na Tabela 4. Na opinião dos pacientes do $H_{A}$, os valores variaram de 4,2(4,0-4,6) para equipe de enfermagem composta por quatro profissionais a 4,9(4,6-5,0) quando o número de auxiliares e técnicos na equipe somavam sete. Já para os pacientes do $H_{B}$ observou-se variação de $3,4(3,2-3,5)$ (quatro profissionais na equipe e três auxiliares/técnicos) a 4,3(3,9-4,6) (turno da noite). Os acompanhantes tiveram a maior satisfação com o turno noturno do $H_{A} 4,8(4,6-4-8)$ e $H_{B} 4,1(4,0-4,5)$ e equipe de enfermagem com três profissionais no $H_{A} 4,8(4,6-5,0)$ e $H_{B}$ com três profissionais e nove respectivamente, $3,8(3,7-4,0)$ e 3,8 $(3,7-3,9)$. 
Tabela 3: Associação do escore global médio de satisfação com as características sociodemográficas e características da hospitalização. São José do Rio Preto, SP, Brasil, 2014

\begin{tabular}{|c|c|c|c|c|}
\hline \multirow{3}{*}{ Características } & \multicolumn{2}{|c|}{ Pacientes } & \multicolumn{2}{|c|}{ Acompanhantes } \\
\hline & $H_{A}(n=200)$ & $H_{B}(n=211)$ & $H_{A}(n=200)$ & $H_{B}(n=211)$ \\
\hline & $M(D P)$ & $M(D P)$ & $\mathrm{M}(\mathrm{IC})$ & $M(I C)$ \\
\hline Hospital & $4,6(0,4)^{*}$ & $3,6(0,4)^{*}$ & $4,5(0,5)^{*}$ & $3,8(0,3)^{*}$ \\
\hline \multicolumn{5}{|l|}{ Sexo } \\
\hline Feminino & $4,5(0,5)^{*}$ & $3,7(0,5)^{*}$ & $4,6(4,5-4,6)^{*}$ & $3,7(3,7-3,8)^{*}$ \\
\hline Masculino & $4,6(0,4)^{*}$ & $3,5(0,3)^{*}$ & $4,5(4,3-4,7)^{*}$ & $3,7(3,7-3,8)^{*}$ \\
\hline \multicolumn{5}{|l|}{ Grau de instrução } \\
\hline Fundamental & $4,6(0,5)^{*}$ & $3,7(0,4)^{*}$ & $4,5(4,4-4,7)^{*}$ & $3,7(3,7-3,8)^{*}$ \\
\hline Médio & $4,6(0,4)^{*}$ & $3,6(0,4)^{*}$ & $4,5(4,5-4,6)^{*}$ & $3,7(3,7-3,8)^{*}$ \\
\hline Superior & $4,7(0,4)$ & $3,5(0,4)^{*}$ & $4,5(4,3-4,7)^{*}$ & $3,8(3,0-4,0)^{*}$ \\
\hline \multicolumn{5}{|l|}{ Renda Mensal } \\
\hline$<1$ & $4,3(0,7)^{*}$ & $3,8(0,5)^{*}$ & $4,6(4,4-4,8)^{*}$ & $3,8(3,7-3,9)^{*}$ \\
\hline $1-3$ & $4,6(0,4)^{*}$ & $3,6(0,4)^{*}$ & $4,5(4,5-4,6)^{*}$ & $3,7(3,7-3,8)^{*}$ \\
\hline $3-5$ & $4,7(0,3)^{*}$ & $3,6(0,4)^{*}$ & $4,5(4,4-4,8)^{*}$ & $3,7(3,6-3,8)^{*}$ \\
\hline$>5$ & $4,6(0,6)^{*}$ & - & $4,7(4,3-5,0)^{*}$ & - \\
\hline \multicolumn{5}{|l|}{ Tipo de internação } \\
\hline SUS & $4,7(0,5)^{*}$ & $3,7(0,4)^{*}$ & $4,5(4,5-4,6)^{*}$ & $3,7(3,7-3,8)^{*}$ \\
\hline Demais operadoras & $4,8(0,2)^{*}$ & $3,6(0,3)^{*}$ & $4,6(4,5-4,7)^{*}$ & $3,8(3,7-3,8)^{*}$ \\
\hline \multicolumn{5}{|l|}{ Modalidade } \\
\hline Clínica & $4,6(0,4)^{*}$ & $3,6(0,4)^{*}$ & $4,5(4,5-4,6)^{*}$ & $3,8(3,7-3,8)^{*}$ \\
\hline Cirúrgica & $4,6(0,5)^{*}$ & $3,6(0,4)^{*}$ & $4,6(4,5-4,7)$ & $3,7(3,7-3,9)^{*}$ \\
\hline \multicolumn{5}{|l|}{ Permanência acompanhante (h) } \\
\hline $1-6$ & - & - & $4,5(4,4-4,7)^{*}$ & $3,7(3,7-3,8) *$ \\
\hline $7-12$ & - & - & $4,6(4,5-4,7)^{*}$ & $3,9(3,7-4,0)^{*}$ \\
\hline $13-18$ & - & - & $4,4(3,9-4,5)^{*}$ & $3,8(3,7-4,0)^{*}$ \\
\hline $19-24$ & - & - & $4,5(4,9-4,7)^{*}$ & $3,8(3,7-4,0)^{*}$ \\
\hline$>24$ & - & - & $4,5(4,4-4,7)^{*}$ & $3,7(3,6-3,7)^{*}$ \\
\hline
\end{tabular}

Tabela 4: Associação entre o escore global médio de satisfação e as características das unidades dos pacientes São José do Rio Preto, SP, Brasil, 2014.

\begin{tabular}{|c|c|c|c|c|}
\hline \multirow{3}{*}{ Características } & \multicolumn{2}{|c|}{ Pacientes } & \multicolumn{2}{|c|}{ Acompanhantes } \\
\hline & $H_{A}(n=200)$ & $H_{B}(n=211)$ & $H_{A}(n=200)$ & $H_{B}(n=211)$ \\
\hline & $M(D P)$ & $M(D P)$ & $M(I C)$ & $M(I C)$ \\
\hline \multicolumn{5}{|l|}{ Turno de trabalho } \\
\hline Manhã & $4,6(4,6-4,7)$ & $3,6(3,5-3,6)$ & $4,5(4,4-4,6)$ & $3,7(3,7-3,9)$ \\
\hline Tarde & $4,7(4,5-4,8)$ & $3,5(3,4-3,5)$ & $4,5(4,4-4,7)$ & $3,7(3,6-3,7)$ \\
\hline Noite & $4,7(4,5-5,0)$ & $4,3(3,9-4,6)$ & $4,8(4,6-4,8)$ & $4,1(4,0-4,5)$ \\
\hline \multicolumn{5}{|l|}{ Técnicos/Auxiliares (N) } \\
\hline 2 & $4,7(4,5-5,0)$ & $3,6(3,5-3,7)$ & $4,8(4,6-5,0)$ & $3,8(3,7-4,0)$ \\
\hline 3 & $4,3(3,9-4,6)$ & $3,4(3,5-3,6)$ & $4,5(4,1-4,7)$ & $3,7(3,5-4,1)$ \\
\hline 7 & $4,9(4,6-5,0)$ & $3,6(3,5-3,7)$ & $4,7(4,3-4,9)$ & $3,8(3,7-3,9)$ \\
\hline 8 & $4,6(4,5-4,7)$ & - & $4,5(4,4-4,6)$ & - \\
\hline 9 & $4,3(4,6-4,7)$ & $3,6(3,8-4,9)$ & $4,5(4,0-4,7)$ & $3,7(3,6-3,8)$ \\
\hline \multicolumn{5}{|l|}{ Enfermeiros (N) } \\
\hline 1 & $4,6(4,5-4,6)$ & $3,5(3,5-3,6)$ & $4,6(4,5-4,7)$ & $3,7(3,7-3,8)$ \\
\hline 2 & $4,7(4,7-4,8)$ & - & $4,5(4,5-4,6)$ & - \\
\hline 3 & $4,6(4,5-4,8)$ & - & $4,5(4,3-4,8)$ & - \\
\hline 4 & $4,4(4,1-4,5)$ & - & $4,1(3,8-4,7)$ & - \\
\hline \multicolumn{5}{|l|}{ Equipe de enfermagem } \\
\hline 3 & $4,7(4,5-5,0)$ & $3,6(3,5-3,7)$ & $4,8(4,6-5,0)$ & $3,8(3,7-4,0)$ \\
\hline 4 & $4,2(4,0-4,6)$ & $3,6(3,5-3,7)$ & $4,5(4,1-4,8)$ & $3,7(3,5-4,1)$ \\
\hline 9 & $4,6(4,5-4,7)$ & $4,1(3,8-4,6)$ & $4,5(4,4-4,7)$ & $3,8(3,7-3,9)$ \\
\hline 10 & $4,7(4,6-4,8)$ & $3,6(3,5-3,6)$ & $4,5(4,4-4,7)$ & $3,7(3,6-3,8)$ \\
\hline
\end{tabular}




\section{DISCUSSÃO}

Este estudo apontou que de maneira geral, tanto os pacientes como seus acompanhantes, em ambas as instituições investigadas, encontravam-se satisfeitos com o atendimento de suas necessidades de cuidados pela equipe de enfermagem. Estes achados ratificam investigações brasileiras. Na primeira, conduzida em unidade de gastrenterologia no interior do estado de São Paulo, constatou-se valor médio de quatro, em uma escala que variava de um a cinco pontos ${ }^{(13)}$. Outro estudo realizado em um hospital universitário, demonstrou que a maior média de satisfação dos pacientes ficou com os atributos da equipe de enfermagem e na satisfação geral ambos com média cinco, em uma escala variando de um a cinco pontos $^{(14)}$. Já em investigação realizada em diversas unidades de internação de um hospital de ensino da região centro-oeste obteve-se $92 \%$ de medianas entre quatro e cinco ${ }^{(15)}$. A satisfação do acompanhante, no entanto, não foi avaliada. Embora as escalas utilizadas, nos estudos acima citados, também apresentassem formato Likert de cinco pontos continham diferentes abordagens da presente pesquisa não incluindo o acompanhante, o que limita, em parte, comparações.

Ocorreram diferenças nos níveis de satisfação entre os hospitais - $H_{A} 4,6(0,4)$ e $H_{B} 3,6(0,4)$. Isto pode ser explicado pelas distintas naturezas, finalidades, portes, tipo de relacionamento com o sistema de saúde e características de constituição jurídica das instituições. $O \mathrm{H}_{\mathrm{A}}$ caracteriza-se como de ensino, atende pacientes de alto nível de complexidade, dispõe de elevado número de profissionais multidisciplinares e tecnologia avançada enquanto $\circ \mathrm{H}_{\mathrm{B}}$ constitui-se em instituição filantrópica para atendimento de menor complexidade. Pequena desigualdade na percepção da satisfação dos pacientes foi mostrada em estudo que comparou dois hospitais de São Paulo, respectivamente, Md 3,8 e 3,5 em uma escala que variava, também, até cinco pontos ${ }^{(12)}$.

Encontrou-se maior percentual de concordância na satisfação dos pacientes referentes ao atendimento das necessidades emocionais e espirituais ( 81 e $82 \%$ ), segurança ( 83 e $67 \%$ ) e atenção (87 e $61 \%$ ) em ambas instituições e informação (83\%) e percepção dolorosa e terapêutica (93\%) no $H_{A}$. Estes achados surpreenderam, pois as áreas de cuidados necessidades emocionais e espirituais ${ }^{(17,19)}$ e segurança ${ }^{(13)}$ têm sido reportadas como as de menor satisfação entre pacientes hospitalizados. Semelhante as demais investigações ${ }^{(13-15,19-21)}$ nenhuma das áreas de cuidados avaliada alcançou o nível desejável de $100 \%$. Eliminações apresentou o menor nível de satisfação (18 e 0,4\%) por parte dos pacientes corroborando outros estudos $^{(13,19)}$.

No olhar dos acompanhantes, as necessidades de segurança (91 e 67\%), percepção dolorosa e terapêutica (89 e 62\%), informação (81 e 53\%) e emocionais e espirituais $\left(76 \% \mathrm{H}_{\mathrm{B}}\right.$ ) foram as mais pontuadas e as áreas de eliminações e integridade física as de menor nível de satisfação. Interessante notar, que diferente de outros contextos, nas instituições investigadas, tanto os pacientes como seus acompanhantes sentem-se seguros com o cuidado realizado. Segurança pode ser definida como a redução ao mínimo aceitável do risco de um dano desnecessário associado com o cuidado de saúde e constitui-se em importante fator de qualidade ${ }^{(20)}$. 
A associação entre o escore médio global de satisfação dos pacientes e acompanhantes e variáveis sociodemográficas e características da hospitalização evidenciou valores significantes $(p<0,05)$ para o hospital investigado, sexo, grau de instrução, renda mensal, tipo e modalidade de internação e permanência do acompanhante junto ao paciente. Variáveis como sexo ${ }^{(13-15,22)}$, nível de escolaridade ${ }^{(12-13,22)}$ e tempo de permanência na unidade ${ }^{(13,22)}$ também têm sido apontadas como influentes na satisfação do paciente hospitalizado. Não foi encontrada relação entre o escore global e idade $(p=0,23)$ e religião $(p=0,64)$. Os pacientes e acompanhantes atendidos pelos convênios de saúde e particulares tiveram nível de satisfação mais elevado com os cuidados prestados pela equipe de enfermagem em relação aos usuários do Sistema Único de Saúde (SUS) excetuando-se os acompanhantes do $H_{B}$.

Do mesmo modo, procurou-se examinar, algumas caraterísticas das unidades de internação e sua relação com o escore global médio de satisfação. Não foram encontradas associações significativas para quantitativo de pessoal de enfermagem em nenhum dos hospitais evidenciando que o número de enfermeiros, técnicos e auxiliares de enfermagem na equipe, neste estudo, pareceu não interferir com o nível de satisfação dos pacientes/acompanhantes quanto ao atendimento de suas necessidades de cuidados. Alta satisfação de pacientes tem sido relacionada com adequada proporção enfermeiro/paciente ${ }^{(23)}$. Contudo, destaca-se também o ambiente de prática profissional ${ }^{(7,23)}$, qualificação da equipe de enfermagem, qualidade da assistência hospitalar e o envolvimento do enfermeiro na participação de tomada de decisão dos cuidados dos pacientes ${ }^{(7)}$ como fatores significativamente associados com a satisfação.

Já no que se refere ao turno de trabalho, ele se constituiu em fator significativo apenas em um dos hospitais $\left(H_{B}\right)$ onde se observou aumento de satisfação no turno da noite. Esta diferença pode, talvez, ser explicada pela qualidade do relacionamento entre os participantes do estudo e a equipe de enfermagem nas unidades investigadas.

Os resultados desta pesquisa limitam-se a percepção e nível de satisfação do paciente e acompanhante com o atendimento de necessidades de cuidados no contexto de duas instituições hospitalares. Torna-se importante destacar que este conceito se encontra atrelado ao ponto de vista individual de cada usuário e limitada a um tempo e espaço.

A satisfação do paciente com o cuidado de enfermagem é reconhecida como um indicador primordial para qualidade e eficácia do processo de cuidar ${ }^{(24-25)}$. Dessa forma, informações sobre a qualidade da atenção recebida pelos pacientes possibilitam propostas de ações para melhoria da qualidade assistencial.

\section{CONCLUSÃO}

Os pacientes e seus acompanhantes mostraram-se, de maneira geral, satisfeitos com o atendimento das necessidades de cuidados, porém algumas áreas importantes apresentaram déficit de satisfação demandando maior atenção da equipe de enfermagem.

A incorporação da percepção e nível de satisfação do acompanhante, neste estudo, acrescenta outro olhar sobre o resultado da assistência e reitera a importância do foco paciente/família para a realização do 
cuidado centrado no paciente. Dessa forma, a identificação das necessidades de cuidados ainda negligenciadas pela equipe de enfermagem sob o olhar de ambos paciente e acompanhante sinalizam o processo de tomada de decisão acerca da gestão do cuidar e a implementação de mudanças na prática.

Estudos futuros em outros cenários assistenciais poderiam aprofundar a visão do acompanhante incluindo, também, a percepção da equipe de enfermagem sobre o atendimento de necessidades cuidativas. Ainda, novos instrumentos poderiam ser construídos ou atualizados para avaliação da assistência prestada aos usuários.

\section{REFERÊNCIAS}

1. Suhonen R, Gustafsson ML, Katajisto J, Välimäki M, Leino-Kilpi H. Nurses' perceptions of individualized care. J Adv Nurs [Internet]. 2010 [acesso em: 15 ago. 2017];66(5):1035-46. Disponível em: http://dx.doi.org/10.1111/j.13652648.2009.05256.x.

2. Hudon C, Fortin M, Haggerty JL, Lambert M, Poitras ME. Measuring patients' perceptions of patient-centered care: a systematic review of tools for family medicine. Ann Fam Med [Internet]. 2011 [acesso em: 15 ago. 2017];9(2):155-64. Disponível em: http://dx.dooi.org/10.1370/afm.1226.

3. Epstein RM, Fiscella K, Lesser CS, Stange KC. Why the nation needs a policy push on patient-centered health care. Health Aff (Millwood) [Internet]. 2010 [acesso em: 15 ago. 2017];29(8):1489-95. Disponível em:

http://dx.doi.org/10.1377/hlthaff.2009.0888.

4. Szareski C, Beuter M, Brondani CM. O familiar acompanhante no cuidado ao adulto hospitalizado na visão da equipe de enfermagem. Rev Gaucha Enferm [Internet]. 2010 [acesso em: 15 ago. 2017];31(4):715-22. Disponível em:

http://dx.doi.org/10.1590/S1983-14472010000400015.

5. Abdelhadi N, Drach-Zahavy A. Promoting patient care: work engagement as a mediator between ward service climate and patient-centred care. J Adv Nurs [Internet]. 2012 [acesso em: 15 ago. 2017];68(6):1276-87. Disponível em: http://dx.doi.org/10.1111/j.1365-2648.2011.05834.x.

6. Suhonen R, Papastavrou E, Efstathiou G, Tsangari H, Jarosova D, Leino-Kilpi $H$, et al. Patient satisfaction as an outcome of individualised nursing care. Scand J Caring Sci [Internet]. 2012 [acesso em: 15 ago. 2017];26(2):372-80. Disponível em: http://dx.doi.org/10.1111/j.1471-6712.2011.00943.x.

7. Aiken LH, Sermeus W, Van den Heede K, Sloane DM, Busse R, McKee M, et al. Patient safety, satisfaction, and quality of hospital care: cross sectional surveys of nurses and patients in 12 countries in Europe and the United States. BMJ [Internet]. 2012 [acesso em: 15 ago. 2017];344:e1717. Disponível em: http://dx.doi.org/10.1136/bmj.e1717. 8. Kalisch BJ, McLaughlin M, Dabney BW. Patient perceptions of missed nursing care. Jt Comm J Qual Patient Saf [Internet]. 2012 [acesso em: 15 ago. 2017];38(4):161-7. Disponível em: https://doi.org/10.1016/S1553-

7250(12)38021-5.

9. Malik SU. Customer Satisfaction, Perceived Service Quality and Mediating Role of Perceived Value. Int J Mark Stud [Internet]. 2012 [acesso em: 15 ago. 2017];4(1):68-76. Disponível em: http://dx.doi.org/10.5539/ijms.v4n1p68. 10. Merkouris A, Andreadou A, Athini E, Hatzimbalasi M, Rovithis M, Papastavrou E. Assessment of patient satisfaction in public hospitals in cyprus: a descriptive study. Health Science Journal [Internet]. 2013 [acesso em: 15 ago.

2017];7(1):28-40. Disponível em: http://www.hsj.gr/medicine/assessment-of-patient-satisfaction-in-public-hospitalsin-cyprus-a-descriptive-study.php?aid=3075.

11. Santos MA, Sardinha AHL, Santos LN. Satisfação dos usuários com os cuidados dos enfermeiros. Rev Gaucha Enferm [Internet]. 2017 [acesso em: 15 ago. 2017];38(1):e57506. Disponível em: http://dx.doi.org/10.1590/1983$\underline{1447.2017 .01 .57506 .}$.

12. Oliveira AML, Guirardello EB. Satisfação do paciente com os cuidados de enfermagem: comparação entre dois hospitais. Rev Esc Enferm USP [Internet]. 2006 [acesso em: 15 ago. 2017];40(1):71-7. Disponível em: http://dx.doi.org/10.1590/S0080-62342006000100010. 
13. Dorigan GH, Guirardello EB. Satisfação do paciente em uma unidade de gastroenterologia. Acta Paul Enferm [Internet]. 2010 [acesso em: 15 ago. 2017];23(4):500-5. Available from: http://dx.doi.org/10.1590/S010321002010000400009.

14. Molina KL, Moura GMSS. A satisfação dos pacientes segundo a forma de internação em hospital universitário. Acta Paul Enferm [Internet]. 2016 [acesso em: 15 ago. 2017];29(1):17-25. Disponível em: http://dx.doi.org/10.1590/1982$\underline{0194201600004 .}$

15. Freitas JS, Silva AEB C, Minamisava R, Bezerra ALQ, Sousa MRG. Quality of nursing care and satisfaction of patients attended at a teaching hospital. Rev Lat Am Enfermagem [Internet]. 2014 [acesso em: 15 ago. 2017];22(3):454-60. Disponível em: http://dx.doi.org/10.1590/0104-1169.3241.2437.

16. Papastavrou E, Andreou P, Tsangari H, Merkouris A. Linking patient satisfaction with nursing care: the case of care rationing - a correlational study. BMC Nurs [Internet]. 2014 [acesso em: 15 ago. 2017];13:26. Disponível em:

http://dx.doi.org/10.1186/1472-6955-13-26.

17. Lyu H, Wick EC, Housman M, Freischlag JA, Makary MA. Patient satisfaction as a possible indicator of quality surgical care. JAMA Surg [Internet]. 2013 [acesso em: 15 ago. 2017];148(4):362-7. Disponível em:

http://dx.doi.org/10.1001/2013.jamasurg.270.

18. Horta WA. Processo de enfermagem. São Paulo: EPU; 1979.

19. Haddad MCL, Évora YDM. Qualidade da assistência de enfermagem: a opinião do paciente internado em hospital universitário público. Cienc Cuid Saude [Internet]. 2008 [acesso em: 15 ago. 2017];7(Supl. 1):45-52. Disponível em: http://dx.doi.org/10.4025/cienccuidsaude.v7i0.6559.

20. Runciman W, Hibbert $P$, Thomson R, Van Der Schaaf T, Sherman H, Lewalle P. Towards an International Classification for Patient Safety: key concepts and terms. Int J Qual Health Care. [Internet]. 2009 [acesso em: 15 ago. 2017];21(1):18-26. Disponível em: http://dx.doi.org/10.1093/intqhc/mzn057.

21. Bezerra ALQ, Silva TO, Paranaguá TTB, Tobias GC, Silva AEBC. Satisfação dos usuários com a segurança na assistência de enfermagem. Revista de enfermagem UFPE on line [Internet]. 2017 [acesso em: 15 ago.

2017];11(2):915-21. Disponível em:

http://www.revista.ufpe.br/revistaenfermagem/index.php/revista/article/view/9961.

22. Findik UY, Unsar S, Sut N. Patient satisfaction with nursing care and its relationship with patient characteristics. Nurs Health Sci [Internet]. 2010 [acesso em: 15 ago. 2017];12(2):162-9. Disponível em:

http://dx.doi.org/10.1111/j.1442-2018.2009.00511.x.

23. Alhusban MA, Abualrub RF. Patient satisfaction with nursing care in Jordan. J Nurs Manag [Internet]. 2009 [acesso em: 15 ago. 2017];17(6):749-58. Disponível em: http://dx.doi.org/10.1111/j.1365-2834.2008.00927.x.

24. American Nurses Association [Internet]. Maryland: American Nurses Association; c2017 [acesso em: 15 ago. 2017]. Disponível em: http://www.nursingworld.org.

25. Acosta AM, Marques GQ, Levandovski PF, Peralta JP, Lima MADS. Satisfação de usuários com cuidados de enfermagem em serviço de emergência: uma revisão integrativa. REME Rev Min Enferm [Internet]. 2016 [acesso em: 15 ago. 2017];20:e938. Disponível em: http://www.dx.doi.org/10.5935/1415-2762.20160008. 